This is the author's version of an article that has been published in the ISIE 2020 proceedings.

Changes were made to this version by the publisher prior to publication.

The final version of record is available at https://dx.doi.org/10.1109/ISIE45063.2020.9152432

\title{
Load Profile Cycle Recognition for Industrial DC Microgrids with Energy Storage Systems
}

\author{
Alexander Männel*, Kevin Müller*, Elias Knöchelmann ${ }^{\dagger}$ and Tobias Ortmaier ${ }^{\dagger}$ \\ *Bosch Rexroth AG, Lohr am Main, Germany, Email: Alexander.Maennel@boschrexroth.de \\ ${ }^{\dagger}$ Gottfried Wilhelm Leibniz Universität Hannover, Institute of Mechatronic Systems, Hannover, Germany
}

\begin{abstract}
Many consumers in production plants like industrial robots or tool machines perform repetitive movements, which lead to a cyclic load demand. However, these load profiles can usually only be roughly estimated at the planning stage. Hence, a subsequent online adaptation of the energy distribution is useful for cases, such as balancing between the charging and discharging amount of energy storage systems to improve those lifetime and usage. This paper presents a novel method of online adaptation for the load distribution of production processes within industrial direct current (DC) microgrids. The online load profile cycle recognition was used to adapt the energy distribution among the sources and loads in the DC microgrid. These sources can be inverters, rectifiers, energy storage systems or decentralized power supply units, such as photo voltaic systems. The approach consists of three major points, the load profile cycle recognition, the load profile analysis and the online adaptation of the energy distribution. This solution was tested in simulation and in experiment with a test rig, that contains an inverter and an energy storage system. The results show, that the load profile will be recognized latest from the third cycle and that the imbalance between charging and discharging amounts of the energy storage is less than $0.6 \%$ for each cycle after adaptation.
\end{abstract}

Index Terms-Cycle Recognition, Industrial DC Microgrid, Energy Storage Systems, Voltage Droop Control, Online Adaptation

\section{INTROdUCTION AND STATE OF THE ART}

Due to an increasing awareness for reducing carbon dioxide emission, energy efficiency becomes continuously more important for industry. In the last few years there has been a growing interest in industrial direct current (DC) microgrids as a new approach for more energy efficient production processes [1]. DC microgrids have been already applied in DC-powered ships or server data centers [2], [3]. In industrial context DC microgrids are supposed to increase the usage of recuperation energy and to reduce conversion losses. Imbalances of the energy supply and demand can be better compensated with DC microgrids than with state of the art alternating current (AC) technology [4]. A crucial point for DC microgrids is the use of energy storage systems (ESS), which ensure a balance between energy supply and demand within the microgrid [5], [6]. The challenge is to achieve a cost-optimized energy distribution between energy suppliers and energy storage systems. Tool and handling machines such as robots mainly perform repetitive movements and therefore, have cyclic power demand (cyclic load profiles) [7]. However, the load profiles of the consumers can usually only be roughly estimated when designing the production plant. Therefore a subsequent online adaptation of the energy distribution depending on certain environmental influences can achieve improvements to the usage of an energy storage. These influences include the current contribution of the decentralized energy supplier, the state of charge (SoC) of the energy storage unit or the latest electricity price.

In order to perform a subsequent online adaptation of the energy distribution within the grid, it is necessary to have knowledge of the cyclic behavior of load profiles of the DC microgrid participants. In this approach, a programmable logic controller (PLC) collects the power consumption data of the individual consumers. The data is then analyzed to identify a cyclic load profile. If a load profile cycle has been detected, the energy distribution is readjusted depending on the desired use case. The use cases are defined by the plant operator and can e.g. include peak shaving or optimization of own consumption. It is also possible to adjust the energy distribution to the latest electricity price. There are already electricity providers which offer variable electricity tariffs for industries [8].

\section{A. System Description}

In Fig. 1 a typical scheme of an industrial DC microgrid is illustrated. The DC microgrid is primarly supplied by the $\mathrm{AC}$ grid through an unidirectional or bidirectional AC-DC

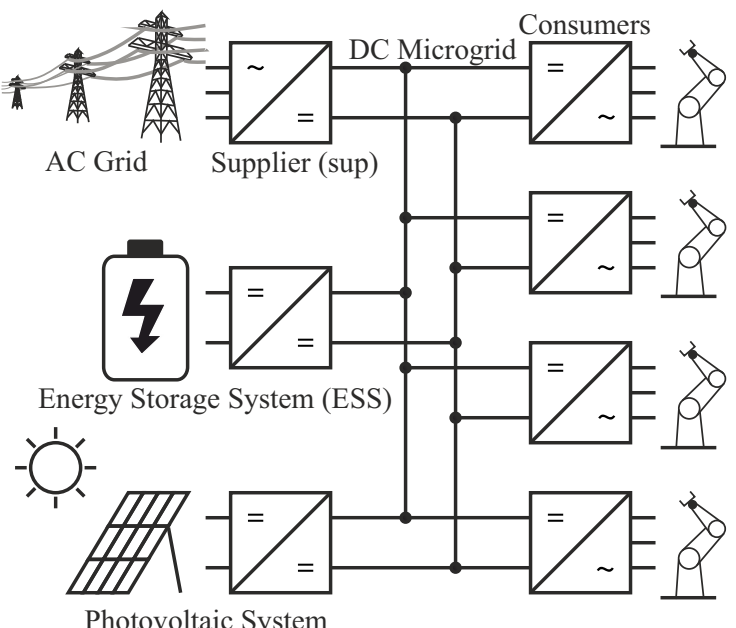

Fig. 1. DC microgrid system with AC-Grid supplier (sup), energy storages systems (ESS), PV System and consumers. 
This is the author's version of an article that has been published in the ISIE 2020 proceedings.

Changes were made to this version by the publisher prior to publication.

The final version of record is available at https://dx.doi.org/10.1109/ISIE45063.2020.9152432

converter (sup). Another important participant for DC microgrids are energy storage systems (ESS), such as lithiumion batteries or super capacitors. They are usually connected with grid-interfacing DC-DC converters. Renewable energy sources, such as photo voltaic systems (PV), become popular for industries to reach carbon neutrality which is targeted by an increasing number of nations [9]. These renewable energy sources can be easily integrated into the DC microgrid with DC-DC converters. Consumers, such as tool and handling machines as well as robots are responsible for the production process. They are driven by three-phase motors, that are supplied by inverters connected to the DC microgrid. In Fig. 1 the consumers are indicated as four industrial robots.

\section{B. Control of DC Microgrids}

Decentralized droop curve control is the most common approach for controlling the converters within DC microgrids. If the impedances of the transmission lines are neglected, it can be assumed that the voltage is equal within the entire grid. Thus, with the help of droop curves, the loads of the consumers can be distributed proportionally to the sources [10]. Therefore, droop curves can be considered as the virtual impedance of the converters [10]. However, droop curve control is inflexible regarding changing constraints, such as the SoC of ESSs or volatile power sources (PV). Thus, the droop curve control could lead to an uncontrolled SoC. On the other hand, flexibility requirements for industrial DC microgrids like connecting or disconnecting grid participants at runtime [4] is also challenging. The different number of participants can result in a wide range of workload situations for the DC microgrid. For this reason, many works [3], [4], [11], [12], propose adaptive droop curve controls with an additional communication link to a superior control unit like a PLC. Even though, this approach requires additional cabling effort and increases complexity due to additional hard- and software, many works use adaptive droop control due to its flexibility [10], [13]-[16]. As the constraints within the microgrid change, the control unit will adapt the droop curve to a new more energy efficient state. For this reason, also this work will be based on this approach.

\section{Cycle Recognition}

This paper is focused on optimizing charging and discharging of energy storages in production processes with cyclic load profiles. In order to identify cyclic load profiles, an algorithm for recognizing cycles is necessary. There are many works in different research fields discussing cycle or pattern recognition. These different applications are among others: medical applications like brain activity, blood pressure or body temperature of a human, economical applications like exchange rates of currencies, or industrial applications [17][20]. In terms of production processes Reger et al. [21] consider cyclic load profile recognition for electric drives of a multiaxial lathe. The recognition of cycles or patterns with different lengths are still a problem [22], thus most existing works finding a cycle require that the approximate length of the time series is known in advance [23]. For example, gait recognition applications use a pre-defined reference signal to recognize a step, [24], [25]. However, the increasing data space capacity makes it possible to analyze patterns with different length [18]-[20]. All these approaches have in common that the data is first generated and then subsequently analyzed in a data exploration [19], [20], [26]. However, the online adaptation of the DC microgrid must be done within in a critical time range. As production processes are usually controlled by PLCs, the online adaptation algorithm must be simple enough for devices with limited computational power and data capacity. This approach also allows to have a reference signal with rough estimation of length and shape. At the time of design, the reference load profile is determined from the motion profile of the machine which is set by the plant operator. However, to the authors best knowledge, few publications [21] are available in the literature that address cycle recognition in terms of production processes for time critical online adjustments. For this reason, in this work a novel method is introduced to adapt the energy distribution of an industrial DC microgrid online, with a load profile cycle recognition. The remainder of this paper is organized as follows: In Sec. II the novel concept of cycle recognition and online adaptation of the energy distribution is introduced. The approach is investigated in simulation with a simple model of a DC microgrid in Sec. III. The test rig for experiment is described in Sec. IV. In Sec. V the simulation is validated and the results are discussed. Sec. VI concludes with a summary.

\section{CONCEPT OF CYCLE RECOGNITION AND ONLINE ADAPTATION OF ENERGY DISTRIBUTION IN DC MICROGRIDS}

The basic concept of cycle recognition and online adaptation to optimize the usage of the ESS within the DC microgrid follows in this section. For this concept a rough estimation of the cyclic load profile is necessary. That is particular because, the algorithm needs a reference point in which time scale $T_{\text {est }}$ it has to look for a cycle period. While the machine is planned by the plant operator, usually simulations or calculations with engineering or sizing tools are carried out, to determine sizes, temperature load or other important parameters of the electrical drives [27]. In this planning state, a rough initial load profile estimate for the machine can be carried out and used for cycle recognition. In operating state, a programmable logic controller or an industrial PC collects the data of the consumer's power consumption $P_{\text {rec }}(t)$ and stores it. On the other hand, the length $T_{\text {est }}$ of the initial load profile estimation is saved as well. With that, the further steps of the concept can be divided into major points:

1) Cycle Recognition which compares the time signal of a reference load profile $P_{\text {ref }}$ with the time signal of the current load profile $P_{\text {rec }}$.

2) Load profile analysis which evaluate the current load profile according to the desired use case. It then determines the power limits for all sources of the DC microgrid (e.g. sup, ESS, PV, etc.). 
This is the author's version of an article that has been published in the ISIE 2020 proceedings.

Changes were made to this version by the publisher prior to publication.

The final version of record is available at https://dx.doi.org/10.1109/ISIE45063.2020.9152432

3) Online adaptation of droop curves follows according to the power limits which were determined in the previous step. With adapted droop curves the energy distribution between the source is updated.

Due to the required flexibility of industrial DC microgrids [4] the connecting or disconnecting of consumers change the cyclic load profile slightly during operation. For this reason, the cycle recognition and online adaptation have to be executed repetitively, to respond on such changes. A state machine is introduced for this approach and visualized in Fig. 2.

\section{A. State Machine}

The cycle recognition consists of the following five states. In first state Record cycle I the loads of the consumers are collected and recorded. This state starts with the operation of the machine. The record data length $P_{\text {rec }}$ depends on the estimated $T_{\text {est }}$ and must be a multiple of it, to ensure that the cycle in the load profile is found. After the record time $T_{\text {rec }}$ has reached a multiple of the estimated time for a cycle period length $T_{\text {est }}$, the state machine switches to the next state Searching cycle to perform a cross-correlation in $\mathbf{C y c R e c}()$ with $T_{\text {est }}$ and $P_{\text {rec }}$. In Sec. II-B the cross-correlation function CycRec() will be explained in detail. Depending on the result of $\operatorname{CycRec}()$ the state machine either switches back to Record cycle I if no cycle was found or it proceeds to Cycle found.

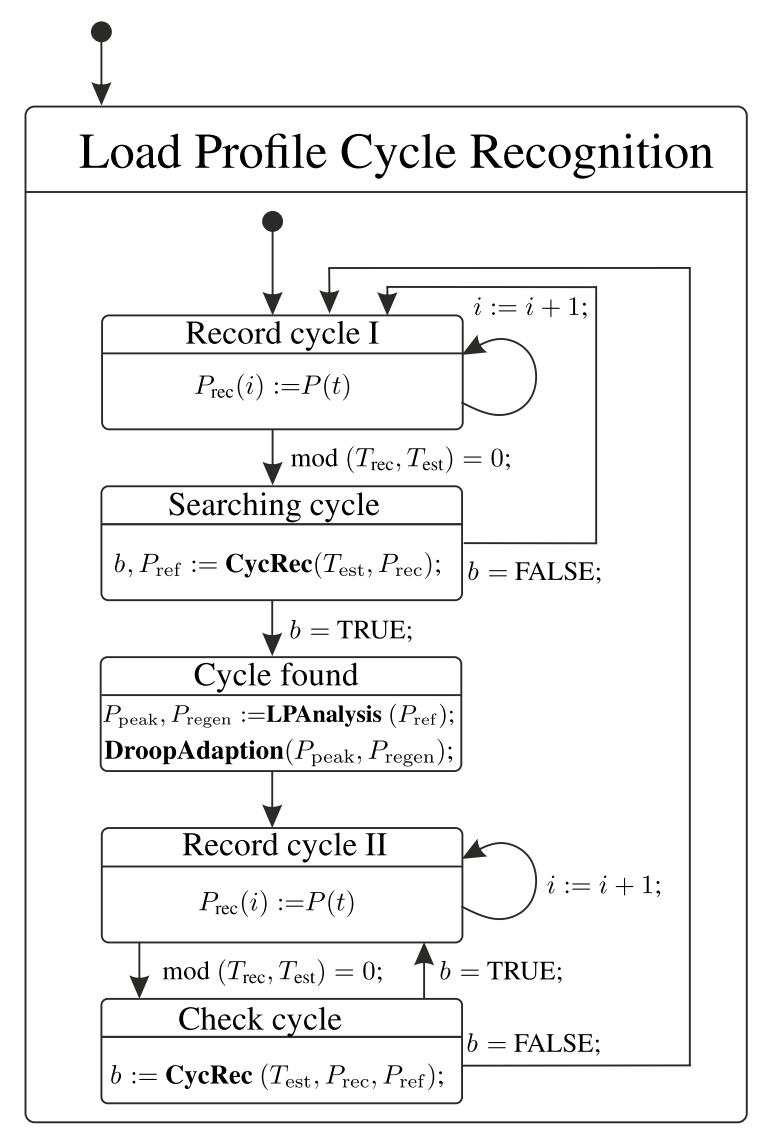

Fig. 2. State machine of the load profile cycle recognition.
In case of the cycle was not found, the algorithm continues collecting load data from the consumers until the record time $T_{\text {rec }}$ has reached the next multiple of the estimated time $T_{\text {est }}$. If CycRec() has found a cycle, the cycle will be used as a reference signal $P_{\text {ref }}$ for the next steps. Then, the functions LPAnalysis() and DroopAdaption() are performed to calculate and implement the new power limits $P_{\text {peak }}$ and $P_{\text {regen }}$ in the Cycle found state. A detailed description of both functions and the power limits can be found in Sec. II-C and Sec. II-D. After the load profile analysis and the droop curve adaptation was successful, the state machine turns into state Record cycle II. This state behaves equal to state Record cycle I. However, after $T_{\text {rec }}$ reached another multiple of $T_{\text {est }}$ the state machine switches to the Check cycle state. In this state, it is checked whether the cyclic load profile still remains or there is a new cycle (e.g. due to a new consumer that is connected to the DC microgrid). CycRec() is executed again, but this time with $P_{\text {rec }}$ and with the additional input, the reference signal $P_{\text {ref }}$ which was obtained in Searching cycle state. If CycRec() find the remaining cycle, the state machines goes back to Record cycle II. If the function hasn't found a remaining cycle, the load condition within the DC microgrid has changed. Thus the whole algorithm needs to start over and the state machine turns back to Record cycle $I$.

\section{B. Cycle Recognition}

The function CycRec() is the main point of the cycle recognition. Input of the function is the recorded power data $P_{\text {rec }}$ from all DC microgrid consumers and the estimated profile length $T_{\text {est }}$. According to the roughly estimated initial load profile, the recorded power data will be separated into two sections. The first section contains at least the latest occurred cycle $n$ with a length of $T_{\text {est }}$, whereas the other section contains the remaining cycles $(n-k)$ with a length of $k \cdot T_{\text {est }}$. Then, segments of latest occurred cycle $n$, are used as signal $x$ and compared with the section which contains the remaining cycles $(n-k)$ (indicated as signal $y)$. The mechanism is visualized in Fig. 3. In order to compare the signals $x$ and $y$ the normalized

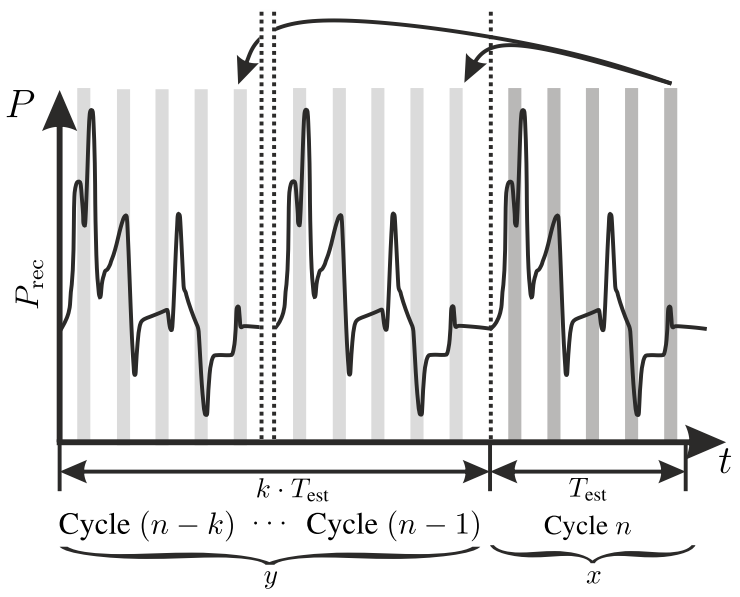

Fig. 3. Segments which are chosen for cycle recognition. 
This is the author's version of an article that has been published in the ISIE 2020 proceedings.

Changes were made to this version by the publisher prior to publication.

The final version of record is available at https://dx.doi.org/10.1109/ISIE45063.2020.9152432

cross-correlation function as

$$
\Phi_{\mathrm{xy}}\{l\}=\frac{\sum_{j=1}^{N} x[j] \cdot y[j+l]}{\sqrt{\sum_{j=1}^{N}(x[j])^{2} \cdot \sum_{j=1}^{N}(y[j+l])^{2}}},
$$

is used [28]. In the next step, the algorithm searches for peaks in the the result of $\Phi_{\mathrm{xy}}\{l\}$. The search for peaks is visualized in Fig. 4. First the mean value and the standard deviation of the cross-correlation result $\Phi_{\mathrm{xy}}$, is calculated as $\overline{\Phi_{\mathrm{xy}}}$ and $\sigma$. Then, the distance of three times standard deviation $3 \cdot \sigma$ from the mean $\overline{\Phi_{x y}}$ is used as the threshold for the peak detection (is indicated as orange line in Fig. 4). The time distance between two peaks (e.g. peak 1 to peak 2 in Fig. 4) is the length $T_{\text {ref }}$ of the cycle. Based on the length $T_{\text {ref }}$ reference cycle $P_{\text {ref }}$ can be extracted from the record data $P_{\text {rec }}$.

\section{Load Profile Analysis}

After the reference load profile cycle $P_{\text {ref }}$ has been obtained, it can be used for analysis depending on the desired use case. In this work, two use cases are considered and introduced in the following:

Optimization of self-consumption (OSC): This means, that all energies from braking events are absorbed by the battery. To prevent an over-charging of the ESS, it has to be discharged at certain times. In this algorithm the ESS is discharged in case of peak loads to maintain a constant SoC of the ESS. However, it must be determined at which power limit $P_{\text {peak }}$ the ESS starts to be discharged in order to keep a balanced SoC. For this reason an energy equilibrium will be calculated by Algorithm 1. The inputs of Algorithm 1, are the recognized load profile $P_{\text {ref }}$ and braking energy $E_{\text {regen }}$ obtained by a numerical integration of the load profile's negative sections. Negative power means an energy flow entering the DC microgrid and positive energy flow means leaving the grid. $E_{\text {regen }}$ is then multiplied by the charge efficiency $\eta_{\text {ch }}$ of the ESS to yield the charging Energy $E_{\text {ch }}$. Then, the highest peak of the load profile $P_{\max }$ and the length of $P_{\text {ref }}$ is determined. After that the iterator $a$ and $\Delta P$ are initialized. Starting from the highest peak demand $P_{\max }$ a loop scans the entire load profile and integrating the sections of the load

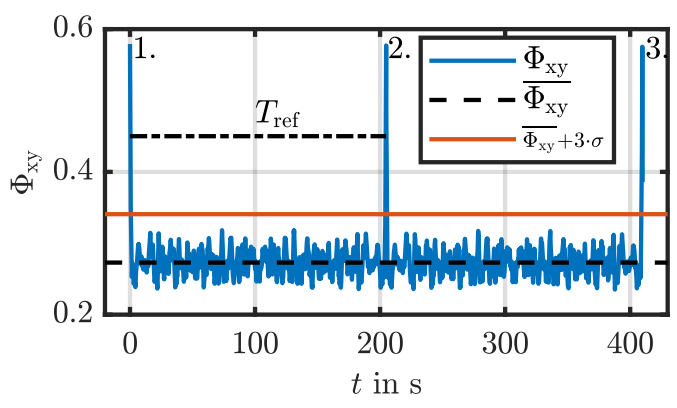

Fig. 4. Determination of $T_{\text {ref }}$ through peak detection.

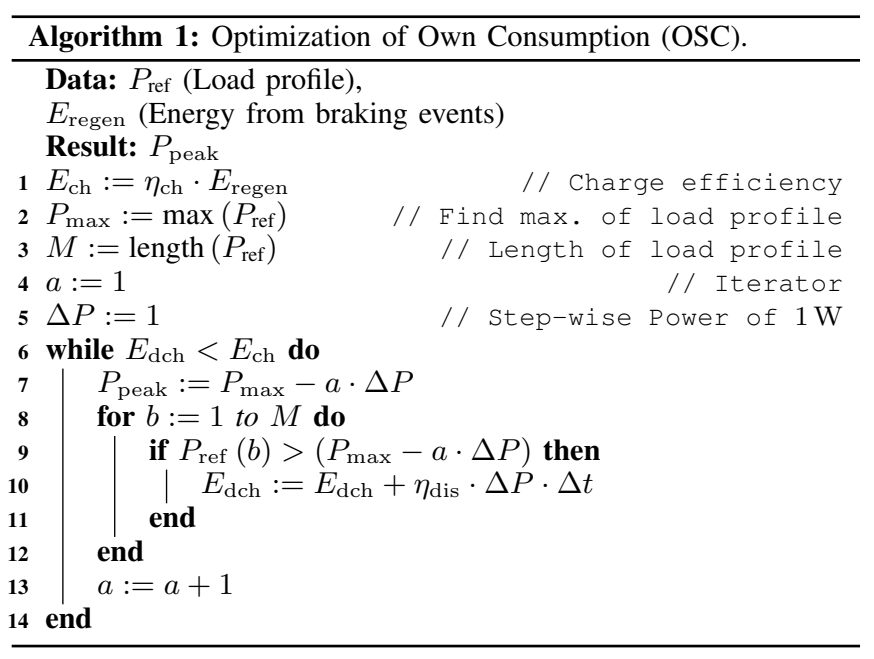

profile which are higher than the current value $P_{\max }-a \cdot \Delta P$. Taking into account the discharge efficiency $\eta_{\text {dis }}$, this results in the discharge energy $E_{\mathrm{dch}}$ of the ESS. This is repeated until the energy equilibrium $E_{\mathrm{dch}}=E_{\mathrm{ch}}$ has been reached - then $P_{\text {peak }}$ is determined. For a better understanding, the result of Algorithm 1 is visualized in Fig. 5 (a). The energy amounts $E_{\text {peak }}$ and $E_{\text {regen }}$ above $P_{\text {peak }}$ and below $P_{\text {regen }}$, are now covered by the ESS, whereas the amount between $P_{\text {peak }}$ and $P_{\text {regen }}$ is contributed by the supplier.

Peak shaving (PS): The maximum load of the supplier must be limited. If the load demand exceeds the maximum load $P_{\text {peak }}$ of the supplier, the ESS starts to support the DC microgrid. The maximum load of the supplier is defined by the plant operator. In order to keep the SoC of the ESS constant over the long-term, the ESS has to be charged whenever the load demand is low. Algorithm 2 computes the point when the ESS must be charged. The energy needed by peak shaving $E_{\text {peak }}$ can be obtained by numerical integration. In most cases the energy needed by peak shaving will exceed the amount of energy which is given by braking processes. Thus, there is still a need to additional charge the battery at low power demand. Algorithm 2 calculates the charging power limit $P_{\text {regen }}$ for the additional charge of the ESS. First, the discharging efficiencies

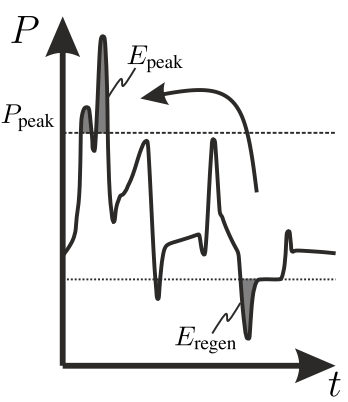

(a)

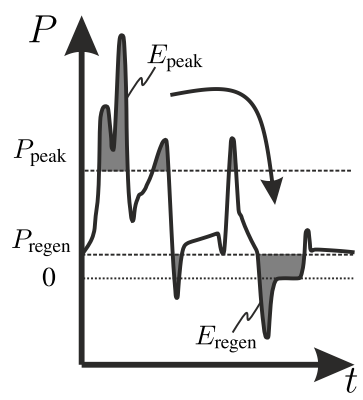

(b)
Fig. 5. Use cases for online adaptation: (a) Alg.1: Optimization of Own Consumption (OSC); (b) Alg. 2: Peak shaving (PS). 
This is the author's version of an article that has been published in the ISIE 2020 proceedings.

Changes were made to this version by the publisher prior to publication.

The final version of record is available at https://dx.doi.org/10.1109/ISIE45063.2020.9152432

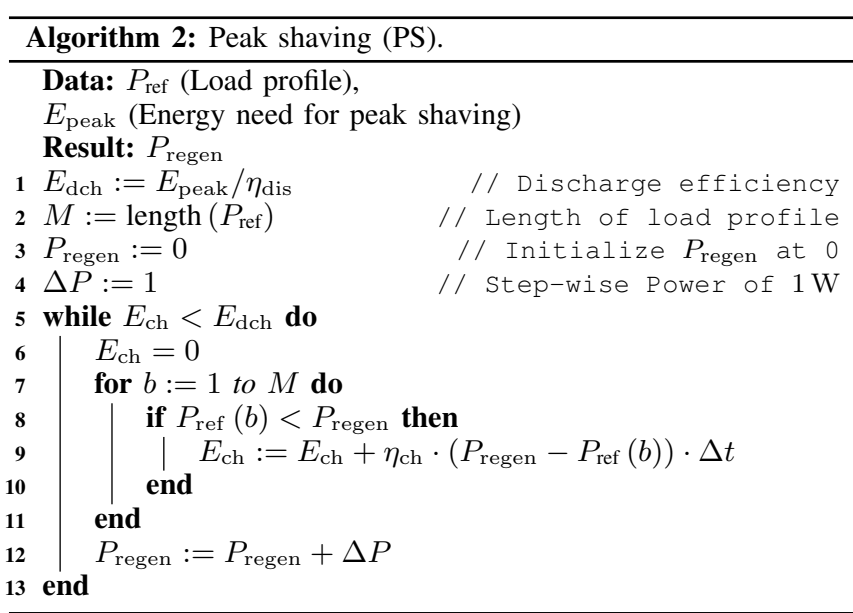

$\eta_{\text {dis }}$ will be taken into account to calculate the discharging amount $E_{\mathrm{dch}}$. Then the iterator $\Delta P$ and the length of the load profile $M$ will be initialized. The algorithm iterates $P_{\text {regen }}$ with $\Delta P$ until the the energy equilibrium $E_{\mathrm{ch}}=E_{\mathrm{dch}}$ is reached. Within that, the algorithm scans the load profile values, which are below the current $P_{\text {regen }}$. If yes, the energy amount $\eta_{\mathrm{ch}} \cdot\left(P_{\text {regen }}-P_{\text {ref }}(b)\right) \cdot \Delta t$ will be added to the charging amount $E_{\mathrm{ch}}$. For a better understanding, the result of Algorithm 2 is visualized in Fig. 5 (b). The energy amounts $E_{\text {peak }}$ and $E_{\text {regen }}$ above $P_{\text {peak }}$ and below $P_{\text {regen }}$, are now covered by the ESS, whereas the amount between $P_{\text {peak }}$ and $P_{\text {regen }}$ is contributed by the supplier.

\section{Online Adaptation of Droop Curves}

In the last step, after $P_{\text {regen }}$ and $P_{\text {peak }}$ were determined as described in Sec. II-C, the droop curves can be adapted. An example of a droop curve sizing for the supplier (blue) and for an ESS (orange) is visualized in Fig. 6. The droop curves have to be sized according to the use case, OSC or PS. In case of a DC microgrid with one supplier and one ESS, the authors suggest the droop curve sizing according to Table I for OSC and Table II for PS. The droop curve of the ESS is set to $\min \left(P_{\text {ref }}\right)$ between $U_{\text {high }}$ and $U_{\text {safe,high in a way, }}$

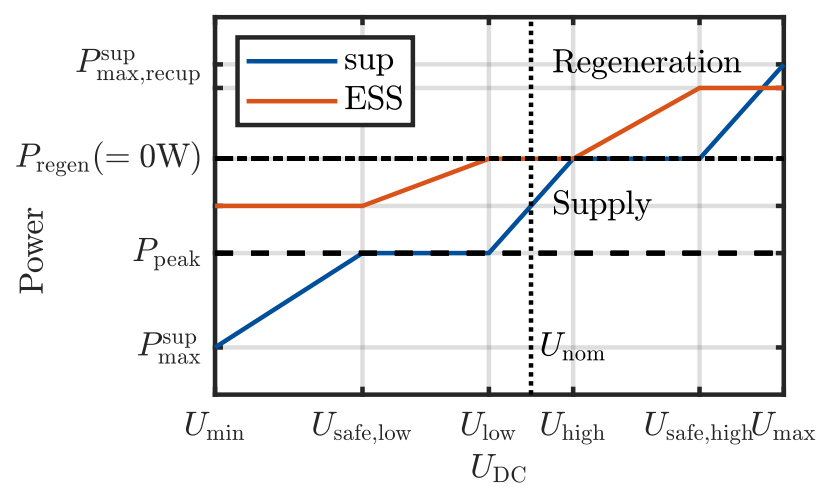

Fig. 6. Droop Curve adaptation for OSC with the use of $P_{\text {peak }}$ and $P_{\text {regen }}$
TABLE I

DETAILED Sizing OF DRoOP CURVES, OSC.

\begin{tabular}{c||c|c|c} 
Power & sup & ESS & Voltage in $V$ \\
\hline$P\left(U_{\text {min }}\right)$ & $P_{\max }^{\text {sup }}$ & $\max \left(P_{\text {ref }}\right)-P_{\text {peak }}$ & 530 \\
\hline$P\left(U_{\text {safe }, \text { low }}\right)$ & $P_{\text {peak }}$ & $\max \left(P_{\text {ref }}\right)-P_{\text {peak }}$ & 570 \\
\hline$P\left(U_{\text {low }}\right)$ & $P_{\text {peak }}$ & 0 & 635 \\
\hline$P\left(U_{\text {nom }}\right)$ & 0 & 0 & 650 \\
\hline$P\left(U_{\text {high }}\right)$ & $P_{\text {regen }}$ & 0 & 675 \\
\hline$P\left(U_{\text {safe }, \text { high }}\right)$ & $P_{\text {regen }}$ & $\min \left(P_{\text {ref }}\right)$ & 750 \\
\hline$P\left(U_{\text {max }}\right)$ & $P_{\text {max }, \text { recup }}^{\text {sup }}$ & $\min \left(P_{\text {ref }}\right)$ & 775
\end{tabular}

TABLE II

Detailed Sizing of Droop CuRVES, PS

\begin{tabular}{c||c|c|c} 
Power & sup & ESS & Voltage in $V$ \\
\hline$P\left(U_{\text {min }}\right)$ & $P_{\max }^{\text {sup }}$ & $\max \left(P_{\text {ref }}\right)-P_{\text {peak }}$ & 530 \\
\hline$P\left(U_{\text {safe, low }}\right)$ & $P_{\text {peak }}$ & $\max \left(P_{\text {ref }}\right)-P_{\text {peak }}$ & 570 \\
\hline$P\left(U_{\text {low }}\right)$ & $P_{\text {peak }}$ & 0 & 635 \\
\hline$P\left(U_{\text {nom }}\right)$ & $-P_{\text {regen }}$ & 0 & 650 \\
\hline$P\left(U_{\text {high }}\right)$ & 0 & $\min \left(P_{\text {ref }}\right)$ & 675 \\
\hline$P\left(U_{\text {safe }, \text { high }}\right)$ & 0 & $\min \left(P_{\text {ref }}\right)$ & 750 \\
\hline$P\left(U_{\text {max }}\right)$ & $P_{\text {max }, \text { recup }}^{\text {sup }}$ & $\min \left(P_{\text {ref }}\right)$ & 775
\end{tabular}

that all energy from regeneration $E_{\text {regen }}$ charges the ESS. As the supplier droop curve provides the base load, it is set to $P_{\text {peak }}$ between $U_{\text {low }}$ and $U_{\text {safe,low }}$. Only in case of load peaks the ESS will be activated. Consequently, the ESS droop curve is set to $\max \left(P_{\text {ref }}\right)-P_{\text {peak }}$. The maximum powers from the supplier are $P_{\max }^{\text {sup }}, P_{\max }^{\text {sup }}$ recup. The idea is to keep a safety margin within the droop curve between $U_{\text {min }}$ and $U_{\text {safe,low }}$, $U_{\text {safe,high }}$ and $U_{\max }$, respectively. Due to the safety margin, the DC microgrid stays stable if the load is higher because of a sudden cyclic load profile change. The voltages in Table I and Table II were used for the experiment.

\section{System Modeling And Simulation}

\section{A. DC-Grid Modeling}

In this work the DC microgrid model, based on [29] and [30], is simplified to an electric network with one capacity $C_{\mathrm{DC}}$ between the DC terminals. If we neglect the transmission line impedances, the sum of the participants' currents $i_{n}$ of the DC microgrid are equal to zero. Then current at the terminals of the capacity $C_{\mathrm{DC}}$, is

$$
i_{\mathrm{CC}}-i_{\mathrm{SUP}}-i_{\mathrm{ESS}}-i_{\mathrm{PV}}-i_{\mathrm{Load}}=0,
$$

where $i_{\text {SUP }}$ is the AC-grid tied supplier's current, $i_{\mathrm{ESS}}$ and $i_{\mathrm{PV}}$ are the current outputs from the DC-DC converters which control the load flow from the ESS and the PV system, respectively. $i_{\text {Load }}$ is the current of all loads demanded by the consumers within the DC microgrid. Since this model neglects the impedances of the transmission lines, it can be assumed that the DC grid voltage $u_{\mathrm{DC}}$ is equal to the capacitance voltage $u_{\mathrm{c}}$ and results to

$$
u_{\mathrm{DC}}(t)=u_{\mathrm{C}}(t)=u_{0}+\frac{1}{C_{\mathrm{DC}}} \int_{t_{0}}^{t} i_{\mathrm{c}}(\tau) \mathrm{d} \tau,
$$


This is the author's version of an article that has been published in the ISIE 2020 proceedings.

Changes were made to this version by the publisher prior to publication.

The final version of record is available at https://dx.doi.org/10.1109/ISIE45063.2020.9152432

with $u_{0}$ as the initial voltage and $t_{0}=0$ as the start time and $t$ as the current time. In the next step, Eq. (3) is transferred from continuous to discrete time integration approximation with the time sample $\Delta t$, which yields

$$
u_{i+1}=u_{i}+\frac{1}{C_{\mathrm{DC}}} i_{i} \Delta t
$$

with $i_{i}$ as the current and $u_{i}$ as the voltage at discrete time step $i$.

\section{EXPERIMENT}

For model validation, a DC microgrid test rig (Fig. 7) was set up which has already been used in [29]-[32]. The test rig contains mains supply (sup) with adaptive droop curve control which is the main supplier to the DC microgrid. The ESS and the DC microgrid are connected via a DCDC converter with adaptive droop curve control. As ESS, a Lithium-ion Battery with $21 \mathrm{kWh}$ energy capacity is used [33]. The total capacity of the DC microgrid is $C_{\mathrm{DC}}=14.2 \mathrm{mF}$, which is the default configuration of the device, that was also used for simulation, [34], [35]. For the complete load representation $P_{\text {ref }}$ a motor inverter with the drive $D_{1}$ in Fig. 7 is used. In order to absorb the energy which is released through the load representation by drive $D_{1}$ a second drive $D_{2}$ supplied by another motor inverter is attached. $D_{1}$ and $D_{2}$ are mechanically connected through a drive shaft. To perform different load profiles, $D_{1}$ is torque controlled and $D_{2}$ is controlled at a constant rotational speed. Both drives were set to a rotational speed of $n_{\mathrm{D}}=1800 \mathrm{~min}^{-1}$, as this is the most efficient speed for these types of drives.

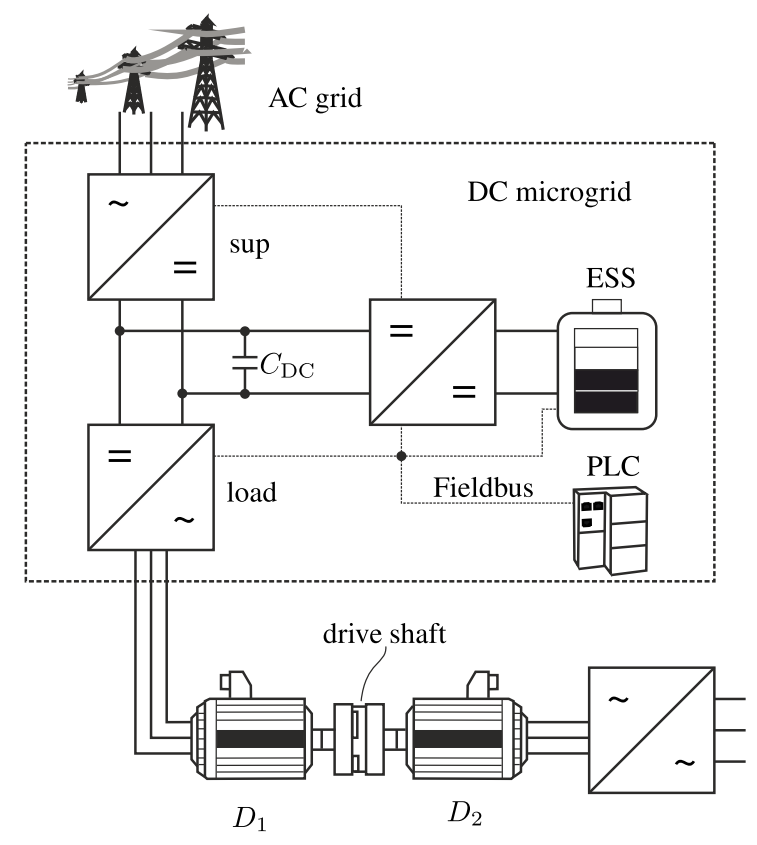

Fig. 7. DC microgrid test rig.
TABLE III

Detailed Energy Storage System.

\begin{tabular}{c||c|c|c|c} 
Scenario & Load profile & Mode & $P_{\text {peak }}$ (only PS) & $T_{\text {est }}$ \\
\hline$I$ & 1 & OSC & - & $115 \mathrm{~s}$ \\
\hline$I I$ & 2 & PS & $7 \mathrm{~kW}$ & $135 \mathrm{~s}$ \\
\hline
\end{tabular}

\section{RESUlTs}

For experiment, two scenarios were performed with two different load profiles, artificially generated by a validated model for computing the power consumption of industrial robots [36]. Load profile 1 consists of two different cycles to show that the algorithm can adapt to different load profiles and small changes of the length (Fig. 8 upper plot). The first cycle has a length of $T_{1}=122 \mathrm{~s}$ and is repeated ten times until $t_{2}=1200 \mathrm{~s}$. The second cycle has a length of $T_{2}=135 \mathrm{~s}$ and is repeated ten times until the end of the experiment. On the right-upper side of Fig. 8 is a zoom, for a better visibility of a single cycle $T_{2}$. The change is also indicated as a black dashed line in Fig. 8. Load profile 2 with a cycle length $T_{3}=133 \mathrm{~s}$ and repeated 15 times is demonstrated with peak shaving (Fig. 10 upper plot). Table III shows the details including the peak shaving limit.

\section{A. Scenario I}

Fig. 8 shows the results of Scenario I. The center plot of Fig. 8 shows the power of the ESS, whereas Fig. 9 shows the adapted droop curves of supplier and ESS, at certain points of
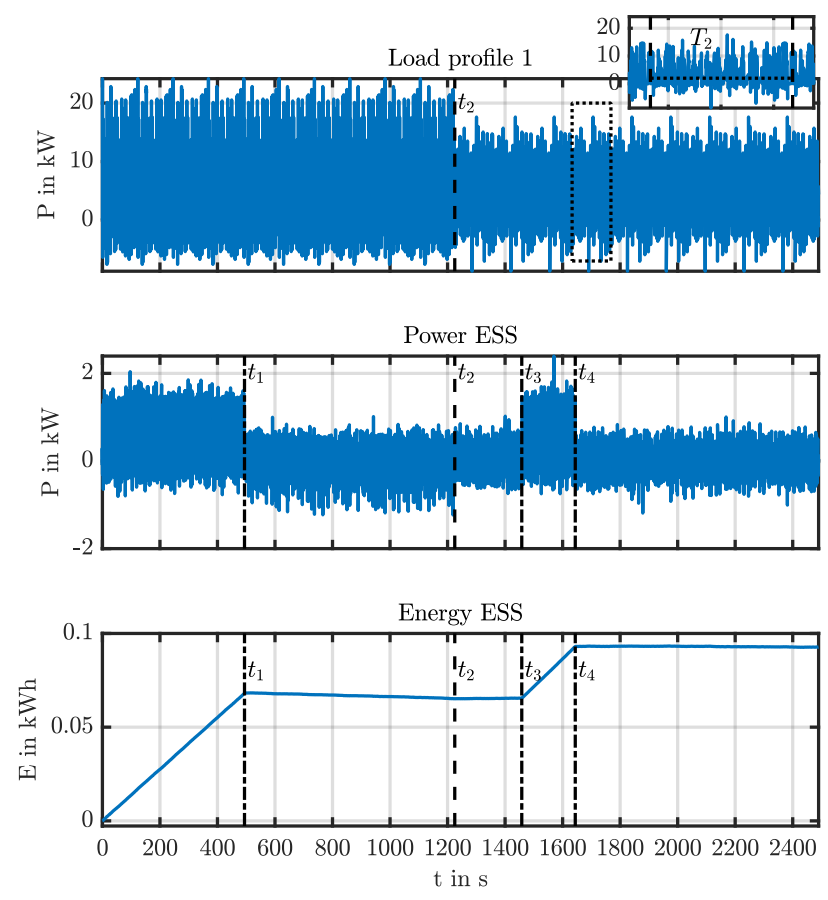

Fig. 8. Scenario I - Upper: Load profile 1; Center: Power ESS; Lower: Energy ESS. 
This is the author's version of an article that has been published in the ISIE 2020 proceedings.

Changes were made to this version by the publisher prior to publication.

The final version of record is available at https://dx.doi.org/10.1109/ISIE45063.2020.9152432
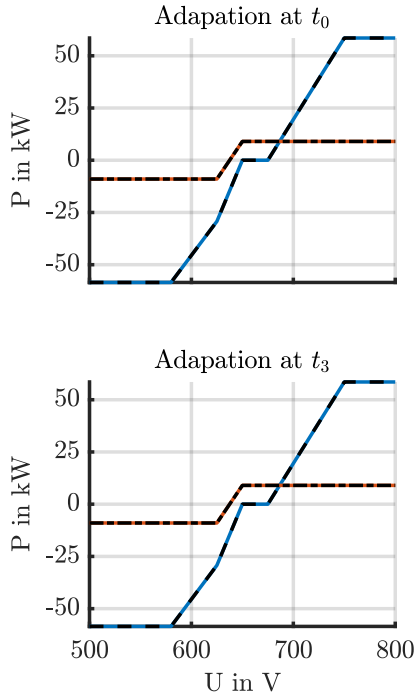

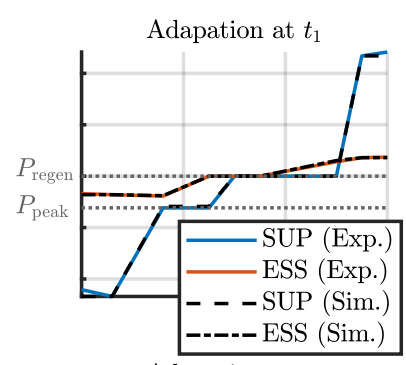

Adapation at $t_{4}$

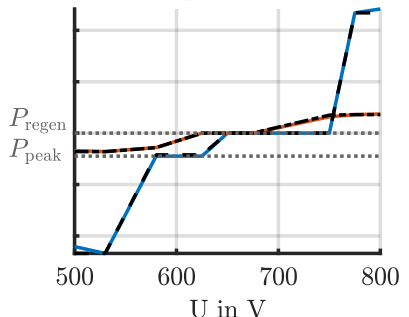

the ESS is tendentially discharged ('Adaptation $t_{0}$ ' Fig. 11). At $t_{1}=580 \mathrm{~s}$ the algorithm recognized the cycle, the droop curve is set to 'Adaptation $t_{1}$ ' (Fig. 11). It can be seen that the supplier still not exceeds the $P_{\text {peak }}$ limit, while the ESS energy consumption is balanced. The imbalance between charging and discharging amounts is merely $\delta=0.40 \%$. The error between droop curve estimation between simulation and experiments is $\epsilon=0.69 \%$

\section{CONCLUSION}

This paper has shown that a cycle recognition with subsequent online adaptation of the energy distribution can keep balance between charging and discharging amounts of an energy storage system. Experiments showed, that the imbalance error is less than $\delta=0.6 \%$ for a cycle period. Thus, the online adaptation improves the balance of the charging and discharging control of the energy storage system. However, due

Fig. 9. Droop curve adaptation in Scenario I

time. Fig 9 also shows a good coverage between the algorithm tested in simulation and in experiment with an maximum error of $\epsilon=0.88 \%$. At $t_{0}=0 \mathrm{~s}$ of Scenario I an initial droop curve, which tendentially charges the ESS, was applied (Fig. 9, 'Adaptation $t_{0}$ '). The initial droop curve is not calculated by any load profile analysis, but was sized by a first rough estimation. At $t_{1}=500 \mathrm{~s}$ the algorithm recognized the cyclic load profile and the droop curve 'Adaptation $t_{1}$ ' in Fig. 9, sized according Sec. II-D, was set. Later on, at $t_{2}=1200 \mathrm{~s}$ the load profile changes to second cyclic profile. This will not be immediately recognized by the algorithm as it takes at least $T_{\text {est }}$. Thus the droop curve 'Adaptation $t_{1}$ ' still remains. At $t_{3}=1450 \mathrm{~s}$, the state machine went through CheckCycle recognized that a cycle has changed. However, the new cycle was not yet long enough recorded to recognize the second cyclic profile. As a consequence, algorithm resets to the initial droop curve (Fig. 9, 'Adaptation $t_{3}$ ') which was also set at $t_{0}$. At $t_{4}=1650 \mathrm{~s}$ the algorithm recognizes the second cyclic load profile and sets the corresponding droop curve 'Adaptation $t_{4}$ ' according Sec. II-D, visualized as in Fig. 9. The lower plot of Fig. 8 shows the energy consumption of the ESS. It shows that the amount of charging and discharging of the ESS is balanced, after a cycle was recognized (between $t_{1}<t<t_{2}$, and $t>t_{4}$ ). The imbalance between charging and discharging for one cycle is significantly low: it is $\delta=0.60 \%$ for the first cyclic profile and second cyclic profile $\delta=0.10 \%$. This imbalance comes from uncertainties of the charging $\eta_{\mathrm{ch}}$ and discharging efficiencies $\eta_{\text {dis }}$ of the ESS.

\section{B. Scenario II}

Scenario II with Load profile 2 and PS as use case is shown in Fig. 10. The maximum peak load $P_{\text {peak }}$ of the supplier was set to $7 \mathrm{~kW}$. The initial droop curve at $t_{0}$ was set so that $P_{\text {peak }}$ is not exceeded by the supplier and that
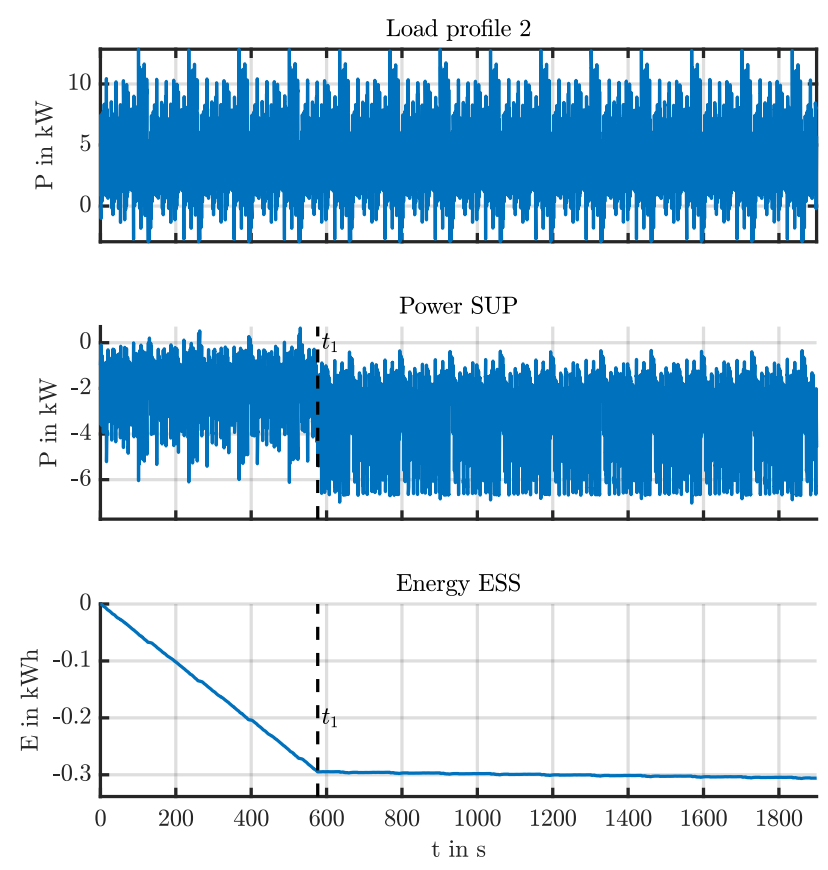

Fig. 10. Scenario II - Upper: Load profile 2; Center: Power sup; Lower: Energy ESS.
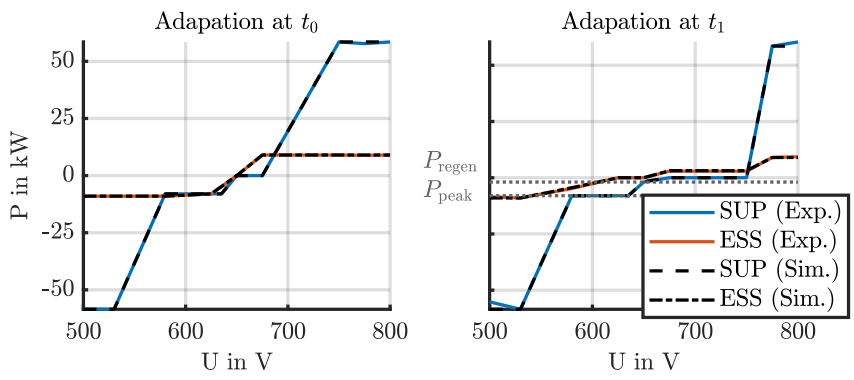

Fig. 11. Droop curve Adaptation in Scenario II. 
This is the author's version of an article that has been published in the ISIE 2020 proceedings.

Changes were made to this version by the publisher prior to publication.

The final version of record is available at https://dx.doi.org/10.1109/ISIE45063.2020.9152432

to the small imbalance which is still left, the energy storage will eventually discharge or charge completely. A combination with a state of charge-based droop curve adaptation which was suggested in [29] and [30] can solve this problem, and will be considered in future work. Furthermore, the algorithm was not tested with break in production or with errors of single consumers. This can be also considered in a future work.

\section{ACKNOWLEDGMENT}

This project was part of the DC-INDUSTRIE research project, funded by the German Federal ministry of Economic Affairs and Energy (BMWi). For further information see: www.dc-industrie.de. The authors would like to thank M. Schappler for revising this paper.

\section{REFERENCES}

[1] K.-P. Simon, "DC Networks in Industrial Production: Research Project DC-INDUSTRIE," Frankfurt am Main, September 2017, accessed Sept. 4, 2018. [Online]. Available: www.dc-industrie.de

[2] L. Ott, Y. Han, B. Wunder, J. Kaiser, F. Fersterra, M. Schulz, and M. Marz, "An Advanced Voltage Droop Control Concept for Grid-Tied and Autonomous DC Microgrids," in IEEE International Telecommunications Energy Conference (INTELEC), 2015, pp. 1-6.

[3] B. Wunder, L. Ott, J. Kaiser, Y. Han, F. Fersterra, and M. Marz, "Overview of Different Topologies and Control Strategies for DC Micro Grids," in IEEE First International Conference on DC Microgrids (ICDCM), 2015, pp. 349-354, DOI: 10.1109/ICDCM.2015.7152067.

[4] H. Borcherding, J. Austermann, T. Kuhlmann, B. Weis, and A. Leonide, "Concepts for a DC Network in Industrial Production," in IEEE ICDCM, 2017, pp. 227-234, DOI: 10.1109/ICDCM.2017.8001049.

[5] W. W. Weaver, R. D. Robinett, G. G. Parker, and D. G. Wilson, "Energy storage requirements of dc microgrids with high penetration renewables under droop control," International Journal of Electrical Power \& Energy Systems, vol. 68, pp. 203-209, 2015. [Online]. Available: http://www.sciencedirect.com/science/article/pii/S0142061514007972

[6] Q. Fu, L. F. Montoya, A. Solanki, A. Nasiri, V. Bhavaraju, T. Abdallah, and D. C. Yu, "Microgrid Generation Capacity Design With Renewables and Energy Storage Addressing Power Quality and Surety," IEEE Transactions on Smart Grid, vol. 3, no. 4, pp. 2019-2027, 2012.

[7] B. Siciliano and O. Khatib, Springer Handbook of Robotics. Cham: Springer International Publishing, 2016.

[8] Next Kraftwerke GmbH, "Variable electricity tariffs for industry \& commerce," 2019, accessed Jan. $8,2019 . \quad$ [Online]. Available: https://www.nextkraftwerke.de/virtuelleskraftwerk/stromverbraucher/variabler-stromtarif

[9] F. Simon, "EU Commission unveils 'European Green Deal': The key points," EURACTIV, 2019, accessed Jan. 21, 2020. [Online]. Available: https://www.euractiv.com/section/energy-environment/news/eucommission-unveils-european-green-deal-the-key-points/

[10] V. Nasirian, A. Davoudi, and F. L. Lewis, "Distributed Adaptive Droop Control for DC Microgrids," in IEEE Applied Power Electronics Conference and Exposition - APEC, 2014, pp. 1147-1152.

[11] T. Dragicevic, X. Lu, J. Vasquez, and J. Guerrero, "DC Microgrids-Part I: A Review of Control Strategies and Stabilization Techniques," IEEE Trans. Power Electron., vol. 31, no. 7, pp. 4876-4891, 2016.

[12] D. A. Schaab, S. Weckmann, T. Kuhlmann, and A. Sauer, "Simulative Analysis of a Flexible, Robust and Sustainable Energy Supply through Industrial Smart-DC-Grid with Distributed Grid Management," Procedia CIRP, vol. 69, pp. 366-370, 2018, DOI: 10.1016/j.procir.2017.11.037.

[13] L. Che and M. Shahidehpour, "DC Microgrids: Economic Operation and Enhancement of Resilience by Hierarchical Control," IEEE Transactions on Smart Grid, vol. 5, no. 5, pp. 2517-2526, 2014.

[14] T. Dragičević, J. M. Guerrero, J. C. Vasquez, and D. Škrlec, "Supervisory Control of an Adaptive-Droop Regulated DC Microgrid With Battery Management Capability," IEEE Trans. Power Electron., vol. 29, no. 2, pp. 695-706, 2014, DOI: 10.1109/TPEL.2013.2257857.

[15] A. Gkountaras, S. Dieckerhoff, and T. Sezi, "Performance analysis of hybrid microgrids applying SoC-adaptive droop control," in 16th European Conference on Power Electronics and Applications, 2014, pp. 1-10, DOI: 10.1109/EPE.2014.6910834.
[16] X. Lu, K. Sun, J. M. Guerrero, J. C. Vasquez, and L. Huang, "State-ofCharge Balance Using Adaptive Droop Control for Distributed Energy Storage Systems in DC Microgrid Applications," IEEE Transactions on Industrial Electronics, vol. 61, no. 6, pp. 2804-2815, 2014.

[17] K. Buza and L. Schmidt-Thieme, Eds., Motif-Based Classification of Time Series with Bayesian Networks and SVMs. Hamburg: Springer, 2008. [Online]. Available: https://rd.springer.com/chapter/10.1007/9783-642-01044-6_9

[18] Esa Alhoniemi, "Unsupervised Pattern Recognition Methods for Exploratory Analysis of Industrial Process Data," PhD Thesis, Helsinki University ofTechnology, Helsinki, 2017.

[19] A. Mueen, E. Keogh, Q. Zhu, S. Cash, and B. Westover, "Exact Discovery of Time Series Motifs," in Proceedings of the 2009 SIAM International Conference on Data Mining, 2009, pp. 473-484.

[20] A. Mueen, "Enumeration of Time Series Motifs of All Lengths," in IEEE 13th International Conference on Data Mining, 2013, pp. 547-556.

[21] A. Reger, C. Oette, A. P. Aires, and R. Steinhilper, "Pattern recognition in load profiles of electric drives in manufacturing plants," in 5th International Electric Drives Production Conference (EDPC). IEEE, 2015, pp. 1-10, DOI: 10.1109/EDPC.2015.7323209.

[22] Y. Gao and J. Lin, "Efficient discovery of time series motifs with large length range in million scale time series," in IEEE International Conference on Data Mining (ICDM), 2017, pp. 1213-1222.

[23] Y. Li, J. Lin, and T. Oates, "Visualizing Variable-Length Time Series Motifs," in Proceedings of the 2012 SIAM International Conference on Data Mining, 2012, pp. 895-906, DOI: 10.1137/1.9781611972825.77.

[24] Y. Ma, R. Fallahzadeh, and H. Ghasemzadeh, "Toward robust and platform-agnostic gait analysis," in IEEE 12th International Conference on Wearable and Implantable Body Sensor Networks (BSN), 2015, pp. 1-6, DOI: 10.1109/BSN.2015.7299366.

[25] Y. Ma, Z. E. Ashari, M. Pedram, N. Amini, D. Tarquinio, K. NouriMahdavi, M. Pourhomayoun, R. D. Catena, and H. Ghasemzadeh, "CyclePro: A Robust Framework for Domain-Agnostic Gait Cycle Detection," IEEE Sensors Journal, vol. 19, no. 10, pp. 3751-3762, 2019, DOI: $10.1109 /$ JSEN.2019.2893225.

[26] C. M. Yeh, N. Kavantzas, and E. Keogh, "Matrix Profile VI: Meaningful Multidimensional Motif Discovery," in IEEE International Conference on Data Mining (ICDM), 2017, pp. 565-574.

[27] Bosch Rexroth AG, "Rexroth IndraSize - Turbo for Drive Sizing," 2019, accessed Sept. 10, 2019. [Online]. Available: https://www.boschrexroth.com/en/xc/products/productsupport/econfigurators-and-tools/indrasize/indrasize-2

[28] J. R. Buck, M. M. Daniel, and A. Singer, Computer explorations in signals and systems using MATLAB, 2nd ed., ser. Prentice Hall signal processing series. Upper Saddle River, N.J.: Prentice Hall and London : Pearson Education, 2002.

[29] A. Männel, S. Tappe, E. Knöchelmann, and T. Ortmaier, "Investigation on an AC Grid Failure Handling of Industrial DC Micro Grids with an Energy Storage," in IEEE International Conference on Industrial Technology (ICIT), 2019, pp. 1710-1716, feb,

[30] A. Männel, E. Knöchelmann, S. Tappe, and T. Ortmaier, "State of Charge Based Characteristic Diagram Control for Energy Storage Systems within Industrial DC Microgrids," in IEEE ICDCM, Matsue, 2019.

[31] E. Knöchelmann, A. Männel, S. Tappe, and T. Ortmaier, "CostOptimized Control of DC Microgrids based on Characteristic Diagrams," in IEEE ICIT, 2019, pp. 1685-1691.

[32] E. Knöchelmann, A. Männel, B. Goetjes, S. Tappe, and T. Ortmaier, "Decentralized Cost-Optimized Fuzzy Control of DC Microgrids," in IEEE ICDCM, Matsue, 2019.

[33] EST-Floattech, "Green Orca High Energy: Technical Brochure," Zandsteen, 2019, accessed Jan. 28, 2020. [Online]. Available: https://www.est-floattech.com/app/uploads/2019/10/Green-Orcatechnical-brochure_oct.pdf

[34] Bosch Rexroth AG, "IndraDrive Power Supply Basic PSB-20 Functions: Application Manual," 2017, accessed Jun. 28, 2018. [Online]. Available: http://www.boschrexroth.com/various/utilities/mediadirectory

[35] Bosch Rexroth AG, "Rexroth IndraDrive ML Universal Inverters HMU05: Instruction Manual," 2017, accessed Jun. 28, 2018. [Online]. Available: http://www.boschrexroth.com/various/utilities/mediadirectory

[36] K. Eggers, E. Knöchelmann, S. Tappe, and T. Ortmaier, "Modeling and experimental validation of the influence of robot temperature on its energy consumption," in IEEE ICIT, 2018, pp. 239-243, DOI: 10.1109/ICIT.2018.8352183. 